\title{
FORMAÇÃO PROFISSIONAL E ATENÇÃO À MULHER VÍTIMA DE VIOLÊNCIA SEXUAL: REVISÃO INTEGRATIVA
}

\author{
PROFESSIONAL TRAINING AND CARE FOR WOMEN VICTIMS OF SEXUAL VIOLENCE: AN INTEGRATIVE REVIEW
}

FORMACIÓN PROFESIONAL Y ATENCIÓN A MUJERES VÍCTIMAS DE VIOLENCIA SEXUAL: UNA REVISIÓN INTEGRADORA

Francisca Alanny Rocha Aguiar ${ }^{1}$

João Victor Lira Dourado 2

Ludmila Fontenele Cavalcanti 3

Luiza Jane Eyre de Souza Vieira 4

Antonio Rodrigues Ferreira Júnior ${ }^{5}$

Raimunda Magalhães da Silva ${ }^{6}$

Palavras-chave:

Violência Contra a Mulher; Violência Sexual; Ensino Superior; Desenvolvimento de Pessoal; Formação Profissional em Saúde.

Keywords:

Violence Against Women; Sex offenses; Education, Higher; Staff Development; Health Human Resource Training.

Palabras clave:

Violencia Contra la Mujer; Delitos Sexuales; Educación Superior; Desarrollo de Personal; Capacitación de Recursos Humanos en Salud.

Submetido: 27 de Mai. de 2020

Aprovado: 20 de Nov. de 2020

Autor(a) para Correspondência: Francisca Alanny Rocha Aguiar R. Antônio Rodrigues Magalhães, 359 Dom Expedito - Sobral, CE CEP: 62050-100 E-mail:alannyrocha2009@hotmail.

com

\section{RESUMO}

Este estudo teve por objetivo identificar a relação entre a formação profissional e atenção à mulher vítima de violência sexual. Trata-se de revisão integrativa realizada entre janeiro e junho de 2018 no site da Coordenação de Aperfeiçoamento de Pessoal de Nivel Superior (CAPES), além das bases Biblioteca Virtual em Saúde (BVS), EBSCO e PubMed. Para a seleção dos estudos, aplicaram-se critérios de inclusão e exclusão, avaliaram-se os títulos, os resumos e as palavras-chave de todas as publicações e ao final se realizou uma leitura dos artigos completos, sendo selecionados 14 estudos para a amostra final. Da análise emergiram 3 categorias: a) Produção do cuidado à mulher em situação de violência sexual; b) Ensino e serviço: campos (des) legitimados para atenção à mulher em situação de violência sexual; e c) Limites na qualificação profissional para atendimento às mulheres em situação de violência sexual. Os profissionais da saúde em atendimento à mulher em situação de violência sexual apresentam dificuldades no reconhecimento dos casos e na prestação de cuidado de modo holístico. Os cursos do setor saúde apresentam disciplinas cujo escopo se detém no indivíduo e nas patologias inerentes às etapas da vida, deixando pouco espaço para abordar condições de saúde que remetem a uma perspectiva integral.

1. Doutora em Saúde Coletiva pela Universidade de Fortaleza (Unifor). E-mail: alannyrocha2009@hotmail.com ORCID: https://orcid.org/0000-0002-6281-4523

2. Mestre em Saúde Pública pela Universidade Federal do Ceará (UFC). E-mail: jvdourado1996@gmail.com 0RCID: https://orcid.org/0000-0002-3269-1286

3. Professora na Universidade Federal do Rio de Janeiro (UFRJ).E-mail: ludmila.ufrj@gmail.com 0RCID: https:// orcid.org/0000-0001-8236-0330

4. Professora na Unifor. E-mail: janeeyre@unifor.br ORCID: https://orcid.org/0000-0002-5220-027X

5. Professor na Universidade Estadual do Ceará (UECE). E-mail: arodrigues.junior@uece.br ORCID: https://orcid. org/0000-0002-9483-8060

6. Professora na Unifor. E-mail: rmsilva@unifor.br 0RCID: https://orcid.org/0000-0001-5353-7520

Certificação de redação científica: E.L.Freire Editora. Edição de texto: Evandro L. Freire. Revisão de provas: Texto definitivo lido e validado pelos autores. 


\section{ABSTRACT}

This study aimed to identify the relationship between professional training and care for women victims of sexual violence. This is an integrative review conducted between January and June 2018 on the website of the Brazilian Coordination for the Improvement of Higher Education Personnel (Coordenação de Aperfeiçoamento de Pessoal de Nivel Superior [CAPES]), in addition to the databases Virtual Health Library (VHL), EBSCO, and PubMed. For selecting the studies, inclusion and exclusion criteria were applied, the titles, abstracts, and keywords of all publications were evaluated, and in the end the full articles were read, then 14 studies were selected for the final sample. Three categories emerged from the analysis: a) Delivery of care for women in situations of sexual violence; b) Teaching and service: (de)legitimated fields of care for women in situations of sexual violence; and c) Professional qualification constraints to deliver care for women in situations of sexual violence. Health professionals delivering care for women in situations of sexual violence show some limitations at the time of recognizing cases and delivering care in a holistic way. The courses in the health sector have subjects whose scope is focused on the individual and on the pathologies inherent to life stages, leaving little space to address health conditions that refer to a comprehensive perspective.

\section{RESUMEN}

Este estudio tuvo como objetivo identificar la relación entre la formación profesional y la atención a mujeres víctimas de violencia sexual. Se trata de una revisión integradora realizada entre enero y junio de 2018 en el sitio web de la Coordinación Brasileña de Perfeccionamiento del Personal de Educación Superior (Coordenação de Aperfeiçoamento de Pessoal de Nível Superior [CAPES]), además de las bases Biblioteca Virtual en Salud (BVS), EBSCO y PubMed. Para la selección de los estudios se aplicaron criterios de inclusión y exclusión, se evaluaron los títulos, los resúmenes y las palabras clave de todas las publicaciones y al final se leyeron los artículos completos, luego se seleccionaron 14 estudios para la muestra final. Del análisis surgieron 3 categorías: a) Prestación de atención a mujeres en situación de violencia sexual; b) Docencia y servicio: campos de (des)legitimados para atención a mujeres en situación de violencia sexual; y c) Limitaciones de calificación profesional para atención a mujeres en situación de violencia sexual. Los profesionales de la salud que prestan atención a mujeres en situación de violencia sexual presentan algunas limitaciones a la hora de reconocer casos y brindar atención de manera integral. Los cursos en el sector de salud tienen asignaturas cuyo alcance se enfoca en el individuo y en las patologías inherentes a las etapas de la vida, dejando poco espacio para abordar condiciones de salud que remiten a una perspectiva integral.

\section{INTRODUÇÃO}

Trata-se a violência como o ato que adota intencionalmente a força, com coação, contra si mesmo, outra pessoa ou contra um grupo ou comunidade, gerando alta probabilidade de resultar em ferimentos, morte, dano psicológico, mau desenvolvimento ou privação $0^{1}$. Assim, todos os dias, milhares de indivíduos são vítimas de violência não fatal. Entre estes se encontram: a) vítimas de agressões que resultam em ferimentos físicos; b) pessoas que sofrem abusos físicos, sexuais e psicológicos; e c) pessoas que se encontram abaixo da linha da pobreza, desprovidas de condições básicas de sobrevivência².

Nas duas últimas décadas, constata-se a dimensão do fenômeno por sua alta incidência, especialmente quando se refere ao público feminino, que comumente se apresenta como grupo vulnerável pela condição suscetível a problemas e danos de saúde ${ }^{3}$. Esse grupo é alvo de preconceitos cristalizados em papéis estereotipados, cujos direitos são desrespeitados na conjuntura da vida social, afetiva, sexual, reprodutiva e do trabalho ${ }^{4}$. Quanto aos agressores, estes podem ter vínculo familiar ou não ou ser desconhecidos ${ }^{5}$.

Entre os tipos de violência contra a mulher se aponta a violência sexual como um fenômeno social e universal, que envolve contextos culturais e posiciona a mulher como sujeito vulnerável. Sua execução se reflete em um exercício de poder no qual a mulher se encontra subordinada aos mandos e desmandos do homem, o que a transforma facilmente em objeto sexual de maridos e pais ${ }^{6}$.

Essa discussão vem ganhando espaço nas duas últimas décadas, tornando-se tema de diferentes campos disciplinares e entidades internacionais, o que demandou a formulação de políticas e programas, 
a organização de práticas e serviços voltados ao seu enfrentamento, a adequação de marcos legais e a construção de modelos de atenção nas diferentes áreas de atuação, a partir da formulação de uma agenda de políticas direcionadas a esse público ${ }^{7}$.

Contudo, apesar do avanço conceitual na organização formal da atenção às mulheres em situação de violência sexual, a maior parte dos serviços não trabalha sob uma perspectiva de gênero, viabilizando práticas pouco emancipatórias, prescritivas e normatizadoras que reforçam a manutenção das relações abusivas ${ }^{8}$.

A ausência de diagnósticos e cuidados adequados às situações de violência sexual decorrem do despreparo e de uma formação calcada no modelo biomédico, que não possibilitam associar as relações entre corpo e sociedade ${ }^{9}$, nos currículos de graduação dos cursos do setor saúde, gerando deficiência na abordagem do tema violência de gênero e suas manifestações. E, mesmo abordando o tema em questão, não se inclui a interseccionalidade gênero, raça/etnia e classe no trato da violência sexual contra a mulher ${ }^{10}$.

Com isso em vista, este estudo teve por objetivo:

- Identificar a relação entre a formação profissional e atenção à mulher vítima de violência sexual.

\section{METODOLOGIA}

Trata-se de revisão integrativa da literatura, caracterizada como método de pesquisa sistemático que viabiliza a produção de conhecimento a partir da reunião e síntese de múltiplas investigações sobre um tópico específico ${ }^{11}$.

0 estudo compreendeu 6 etapas metodológicas: a) identificação do tema e definição da questão norteadora; b) estabelecimento dos critérios de inclusão e exclusão de estudos; c) identificação das pesquisas pré-selecionadas e selecionadas; d) categorização das investigações incluídas; e) análise e interpretação das informações; e f) apresentação da revisão/síntese do conhecimento ${ }^{12}$.

Elaborou-se a seguinte pergunta de pesquisa:

- 0 que a literatura aborda sobre a relação entre formação profissional e atenção à mulher vítima de violência sexual?

A construção da pergunta envolveu a estratégia PICO ( $P$ - população: profissionais da saúde; I - intervenção/interesse/variável independente: educação sobre violência sexual; C - comparação/ variável dependente: formação profissional; 0 resultados: atenção qualificada à mulher vítima de violência sexual).

Para a operacionalização do estudo, selecionaramse os seguintes termos controlados em português e inglês, disponiveis na base Descritores em Ciências da Saúde (DeCS): a) violência contra a mulher (violence against women); b) violência sexual (sex offenses); c) ensino superior (education, higher); d) desenvolvimento de pessoal (staff development); e) educação médica (education, medical); f) formação profissional em saúde (health human resource training); e g) educação em enfermagem (education, nursing). Para o cruzamento, empregou-se o operador booleano and e realizou-se a combinação dos descritores "violência contra a mulher (violence against women)" e "violência sexual (sex offenses)" adicionada a mais outro termo, totalizando 5 cruzamentos (Tabela 1 ).

0 levantamento bibliográfico foi realizado entre janeiro e junho de 2018, simultaneamente, por 2 pesquisadores, em 4 sites: a) Coordenação de Aperfeiçoamento de Pessoal de Nivel Superior (CAPES); b) Biblioteca Virtual em Saúde (BVS); c) EBSCO; e d) PubMed.

Os critérios de inclusão adotados foram: a) artigos disponiveis em formato eletrônico; b) artigos publicados em português, inglês e espanhol; e c) artigos publicados entre 2013 e 2017.

Já os critérios de exclusão foram: a) documentos indisponiveis; b) documentos duplicados; c) monografias, dissertações, teses, editoriais, matérias jornalísticas, análise conjuntural, discussão teórica de conceitos, artigos não originais (resenhas, comentários); d) manuscritos com amostras de outros grupos que não mulheres; e e) estudos secundários.

0 cruzamento originou 109.751 artigos; 108.114 deles foram excluídos, resultando em 1.637 estudos elegíveis. Após a leitura dos títulos, dos resumos e

\section{... a maior parte dos serviços não trabalha sob uma perspectiva de gênero...}


das palavras-chave foram excluídos 1.619 por não apresentarem relação com o tema, restando 14 artigos que articulavam de algum modo a formação profissional à atenção à mulher vítima de violência sexual (Tabela 1 ).

Nesse processo: a) 4 artigos abordavam a formação de profissionais da saúde e a assistência a mulheres em situação de violência sexual; b) 9 discorriam sobre a atuação profissional junto às mulheres que vivenciam diferentes violências de gênero, incluindo a violência sexual; c) 3 apontavam o hiato existente na formação em campos de atuação profissional, como os serviços que atendem mulheres em situação de violência sexual; e d) 2 discutiam estratégias de atenção à saúde junto a mulheres vítimas de violência, incluindo vítimas de violência sexual.

Tabela 1 - Distribuição dos artigos selecionados nos sites. Sobral, 2019

\begin{tabular}{|c|c|c|c|c|c|c|c|c|}
\hline \multirow{2}{*}{ Cruzamentos } & \multicolumn{3}{|c|}{ Descritores em inglês } & \multicolumn{4}{|c|}{ Descritores em português } & \multirow{2}{*}{ Total } \\
\hline & BVS & CAPES & EBSCO & PUBMED & BVS & CAPES & EBSCO & \\
\hline $\begin{array}{l}\text { "Violência contra a } \\
\text { mulher" and "violência } \\
\text { sexual" and "ensino } \\
\text { superior" }\end{array}$ & 0 & 1 & 0 & 0 & 0 & 1 & 2 & 4 \\
\hline $\begin{array}{l}\text { "Violência contra } \\
\text { a mulher" and } \\
\text { "violência sexual" and } \\
\text { "desenvolvimento de } \\
\text { pessoal" }\end{array}$ & 0 & 0 & 0 & 0 & 1 & 0 & 0 & 1 \\
\hline $\begin{array}{l}\text { “Violência contra a } \\
\text { mulher" and "violência } \\
\text { sexual" and "educação } \\
\text { médica" }\end{array}$ & 1 & 0 & 1 & 0 & 1 & 0 & 1 & 4 \\
\hline $\begin{array}{l}\text { "Violência contra a } \\
\text { mulher" and "violência } \\
\text { sexual" and "formação } \\
\text { profissional em saúde" }\end{array}$ & 0 & 0 & 0 & 0 & 0 & 0 & 0 & 0 \\
\hline $\begin{array}{l}\text { "Violência contra a } \\
\text { mulher" and "violência } \\
\text { sexual" and "educação em } \\
\text { enfermagem" }\end{array}$ & 1 & 1 & 0 & 0 & 0 & 1 & 1 & 4 \\
\hline TOTAL & 2 & 2 & 1 & 0 & 2 & 2 & 4 & 14 \\
\hline
\end{tabular}

Fonte: Elaborada pelos autores.

Após a leitura exaustiva dos manuscritos, utilizou-se um instrumento construído pelos autores para documentar e sumarizar o título das publicações, autores, ano, periódico das produções, cenário e objetivo dos estudos, quantidade de participantes, metodologia, mensuração de variáveis, métodos de análise, teoria ou conceito, principais resultados e conclusões dos artigos selecionados na etapa anterior.

$\mathrm{Na}$ análise e interpretação das informações, buscou-se estabelecer consensos e dissensos, e situar a relação entre os achados e a questão norteadora da pesquisa.

\section{RESULTADOS E DISCUSSÃO}

0 ano de publicação dos estudos variou entre 2014 e 2016. Os estudos incluídos na amostra foram, em sua maioria, desenvolvidos no Brasil. Quanto ao delineamento metodológico, a abordagem qualitativa foi prevalente, totalizando 9 estudos (Quadro 1). 
Quadro 1 - Caracterização dos estudos selecionados. Sobral, 2019

\begin{tabular}{|c|c|c|c|c|}
\hline $\mathrm{N}$ & Autoria/ano & País & Objetivo & Resultados \\
\hline I & $\begin{array}{c}\text { Conn et al. } \\
(2014)^{13}\end{array}$ & Canadá & $\begin{array}{l}\text { Identificar lacunas } \\
\text { de conhecimento, } \\
\text { barreiras percebidas e } \\
\text { capacitadores para a } \\
\text { prática de triagem IPV } \\
\text { na clínica ortopédica. }\end{array}$ & $\begin{array}{l}\text { Aponta uma deficiência de conhecimento dos } \\
\text { residentes quanto à violência por parceiro } \\
\text { íntimo, falta de tempo e privacidade para } \\
\text { esse atendimento e a crença de que a } \\
\text { assistência à mulher vitimada não é papel do } \\
\text { cirurgião. }\end{array}$ \\
\hline II & $\begin{array}{l}\text { Yildiz T et al. } \\
\qquad(2014)^{14}\end{array}$ & Turquia & $\begin{array}{l}\text { Determinar as atitudes } \\
\text { mais relevantes tomadas } \\
\text { pelos enfermeiros } \\
\text { diagnosticando } \\
\text { violência. }\end{array}$ & $\begin{array}{c}\text { Os enfermeiros de emergência foram } \\
\text { identificados com falta de informação no } \\
\text { pré-treinamento sobre as mulheres que } \\
\text { sofreram violência, e após o treinamento } \\
\text { eles apresentaram um entendimento mais } \\
\text { amplo. }\end{array}$ \\
\hline III & $\begin{array}{l}\text { Vieira et al. } \\
\qquad(2016)^{15}\end{array}$ & Brasil & $\begin{array}{l}\text { Analisar a utilização de } \\
\text { protocolos na atenção } \\
\text { à saúde de mulheres em } \\
\text { situação de violência } \\
\text { sexual sob a ótica de } \\
\text { profissionais em duas } \\
\text { capitais brasileiras. }\end{array}$ & $\begin{array}{l}\text { Observa-se fragilidade da qualificação } \\
\text { profissional para atendimento às mulheres } \\
\text { em situação de violência sexual, decorrente } \\
\text { da limitada abordagem sobre esse tema } \\
\text { durante a graduação em saúde, agravada } \\
\text { pela falta de treinamento nos serviços; } \\
\text { o que contribui para a invisibilidade da } \\
\text { demanda e para a assistência adequada. }\end{array}$ \\
\hline IV & $\begin{array}{l}\text { Almeida et } \\
\text { al. }(2014)^{16}\end{array}$ & Brasil & $\begin{array}{l}\text { Identificar e } \\
\text { compreender os } \\
\text { diferentes elementos do } \\
\text { processo de trabalho na } \\
\text { assistência à saúde da } \\
\text { mulher em situação de } \\
\text { violência de gênero. }\end{array}$ & $\begin{array}{c}\text { Evidenciou a invisibilidade da violência no } \\
\text { serviço e o desconhecimento da categoria } \\
\text { gênero e da sua complexidade, indicando a } \\
\text { necessidade da reorientação da formação } \\
\text { dos profissionais da saúde no sentido de } \\
\text { investigar e agir diante da violência de } \\
\text { gênero. }\end{array}$ \\
\hline V & $\begin{array}{c}\text { Gomes et al. } \\
(2014)^{17}\end{array}$ & Brasil & $\begin{array}{c}\text { Compreender o } \\
\text { significado do apoio } \\
\text { psicológico à mulher em } \\
\text { situação de violência } \\
\text { conjugal, no âmbito } \\
\text { da Estratégia Saúde da } \\
\text { Família (ESF). }\end{array}$ & $\begin{array}{c}\text { Os profissionais apontaram seu despreparo } \\
\text { profissional para identificar as mulheres que } \\
\text { vivenciaram a violência conjugal. }\end{array}$ \\
\hline VI & $\begin{array}{l}\text { Porto et al. } \\
(2014)^{18}\end{array}$ & Brasil & $\begin{array}{l}\text { Analisar as percepções } \\
\text { dos profissionais da } \\
\text { ESF a respeito do } \\
\text { enfrentamento da } \\
\text { violência doméstica e } \\
\text { sexual. }\end{array}$ & $\begin{array}{c}\text { 0s participantes reconheceram a } \\
\text { complexidade da violência doméstica e } \\
\text { sexual, mas evidenciaram inabilidade para a } \\
\text { identificação das vítimas e despreparo para } \\
\text { a abordagem e o acompanhamento dos casos. } \\
\text { Constatou-se a importância de incluir o tema } \\
\text { durante o período de formação acadêmica } \\
\text { e implementar políticas de educação } \\
\text { permanente. }\end{array}$ \\
\hline
\end{tabular}




\begin{tabular}{|c|c|c|c|c|}
\hline$N$ & Autoria/ano & Pais & Objetivo & Resultados \\
\hline VII & $\begin{array}{l}\text { Dourado e } \\
\text { Noronha } \\
(2015)^{19}\end{array}$ & Brasil & $\begin{array}{l}\text { Estimar a prevalência } \\
\text { de lesões na face, } \\
\text { cabeça e pescoço de } \\
\text { mulheres agredidas pelo } \\
\text { parceiro; apreender os } \\
\text { significados na visão } \\
\text { das vítimas das marcas } \\
\text { originárias da violência; } \\
\text { e investigar questões } \\
\text { acerca do cuidado em } \\
\text { saúde. }\end{array}$ & $\begin{array}{l}\text { A violência contra a mulher ainda configura } \\
\text { um grande desafio para os profissionais } \\
\text { da saúde. Entre as limitações elencadas } \\
\text { estão: despreparo ou falta de capacitação } \\
\text { adequada; resistências culturais para } \\
\text { abordar a questão com as pacientes; e } \\
\text { priorização do modelo biologicista, em } \\
\text { detrimento dos preceitos da integralidade. }\end{array}$ \\
\hline VIII & $\begin{array}{l}\text { Cordeiro et } \\
\text { al. }(2015)^{20}\end{array}$ & Brasil & $\begin{array}{l}\text { Identificar a relação } \\
\text { entre formação } \\
\text { profissional e } \\
\text { notificação da violência } \\
\text { contra a mulher na ESF. }\end{array}$ & $\begin{array}{l}\text { A violência contra a mulher não foi abordada } \\
\text { na graduação e pós-graduação da maioria } \\
\text { dos profissionais; contudo, para os demais } \\
\text { a abordagem deu-se de forma superficial e } \\
\text { pontual. }\end{array}$ \\
\hline IX & $\begin{array}{l}\text { Santos J et } \\
\text { al. }(2014)^{21}\end{array}$ & Brasil & $\begin{array}{l}\text { Descrever a assistência } \\
\text { à mulher vítima da } \\
\text { violência em unidades } \\
\text { de saúde em de Vitória } \\
\text { da Conquista-BA e no } \\
\text { contexto de trabalho das } \\
\text { coordenadoras dessas } \\
\text { unidades. }\end{array}$ & $\begin{array}{l}\text { A maioria dos profissionais declararam } \\
\text { conhecer a política de proteção à mulher, } \\
\text { mas indicaram dificuldades para colocá-la } \\
\text { em prática. Considerado que o tema é pouco } \\
\text { pesquisado no setor saúde, faz-se necessário } \\
\text { qualificar os profissionais, especialmente } \\
\text { em termos de conhecimento e domínio das } \\
\text { leis e dos decretos que visam a assegurar } \\
\text { programas e ações de proteção às mulheres e } \\
\text { de organização do sistema de saúde. }\end{array}$ \\
\hline$x$ & $\begin{array}{l}\text { Bezerra et } \\
\text { al. }(2016)^{22}\end{array}$ & Brasil & $\begin{array}{c}\text { Analisar os sentidos } \\
\text { atribuídos por } \\
\text { profissionais da saúde } \\
\text { aos conceitos, causas } \\
\text { e repercussões da } \\
\text { violência sexual contra a } \\
\text { mulher. }\end{array}$ & $\begin{array}{l}\text { Os profissionais apresentam dificuldades } \\
\text { em trabalhar com o tema, remetendo a uma } \\
\text { falta de reflexão ou até de conhecimento do } \\
\text { que seja violência; e o não questionamento, } \\
\text { aliado a uma falta de capacitação que os } \\
\text { ajude a pensar, torna-se uma situação } \\
\text { crítica para os pacientes e a reprodução da } \\
\text { violência. }\end{array}$ \\
\hline$X I$ & $\begin{array}{l}\text { Schaffir et } \\
\text { al. }(2014)^{23}\end{array}$ & $\begin{array}{l}\text { Estados } \\
\text { Unidos } \\
\text { da } \\
\text { América } \\
\text { (EUA) }\end{array}$ & $\begin{array}{l}\text { Identificar como os } \\
\text { temas psicossociais } \\
\text { relacionados à saúde da } \\
\text { mulher são ensinados e } \\
\text { avaliados no programa } \\
\text { de obstetrícia/ } \\
\text { ginecologia das } \\
\text { escolas médicas norte- } \\
\text { americanas. }\end{array}$ & $\begin{array}{l}\text { No total, } 48 \% \text { das escolas incluem } \\
\text { treinamento formal em transtornos do } \\
\text { humor relacionados à gravidez; } 58 \% \text { incluem } \\
\text { sindrome pré-menstrual/transtorno pré- } \\
\text { menstrual; } 51 \% \text { incluem disfunção sexual } \\
\text { feminina; e } 79 \% \text { incluem a violência contra } \\
\text { as mulheres. Além disso, } 12 \% \text { das escolas } \\
\text { não listaram nenhum desses tópicos como } \\
\text { ensinado. Todos, exceto } 3 \text { dos diretores, } \\
\text { concordaram que os tópicos psicossociais são } \\
\text { importantes. }\end{array}$ \\
\hline
\end{tabular}




\begin{tabular}{|c|c|c|c|c|}
\hline $\mathbf{N}$ & Autoria/ano & País & Objetivo & Resultados \\
\hline XII & $\begin{array}{l}\text { Baragatti et } \\
\text { al. }(2014)^{24}\end{array}$ & Brasil & $\begin{array}{l}\text { Descrever a experiência } \\
\text { da inserção e do } \\
\text { desenvolvimento de uma } \\
\text { disciplina "Violência, } \\
\text { Saúde e Gênero" em um } \\
\text { curso de graduação em } \\
\text { enfermagem. }\end{array}$ & $\begin{array}{c}\text { A disciplina específica sobre violência } \\
\text { possibilitou que os alunos falassem sobre } \\
\text { o assunto, permitindo amplas discussões, } \\
\text { sendo que muitos relataram experiências } \\
\text { com suas diversas tipologias e suas } \\
\text { diferentes naturezas, vivenciadas por eles ao } \\
\text { longo da vida. }\end{array}$ \\
\hline XIII & $\begin{array}{c}\text { Silva et al. } \\
(2015)^{25}\end{array}$ & Brasil & $\begin{array}{l}\text { Analisar os limites das } \\
\text { práticas de assistência } \\
\text { às mulheres em situação } \\
\text { de violência, fornecidas } \\
\text { pelas equipes da ESF na } \\
\text { rede de saúde. }\end{array}$ & $\begin{array}{l}\text { Constatou-se o despreparo dos profissionais } \\
\text { para trabalhar com o tema violência contra a } \\
\text { mulher e na perspectiva de rede, por falta de } \\
\text { qualificação, e a extensão dessa inaptidão } \\
\text { dos trabalhadores da delegacia de polícia. } \\
\text { As práticas são focadas na queixa-crime, em } \\
\text { protocolos rígidos, e o que escapa da rotina } \\
\text { é tratado com descaso, juízo de valor e com } \\
\text { a acepção de que o problema é de natureza } \\
\text { privada. }\end{array}$ \\
\hline XIV & $\begin{array}{l}\text { Nielson et } \\
\text { al. }(2015)^{26}\end{array}$ & EUA & $\begin{array}{l}\text { Revelar a diferença nas } \\
\text { atitudes em relação aos } \\
\text { pacientes atendidos } \\
\text { por enfermeiros de } \\
\text { emergência treinados } \\
\text { para ser examinadores } \\
\text { da violência sexual } \\
\text { e aqueles sem } \\
\text { treinamento. }\end{array}$ & $\begin{array}{c}\text { Houve diferença significativa nas atitudes } \\
\text { em relação aos pacientes que foram } \\
\text { atendidos pelos enfermeiros treinados e } \\
\text { àqueles sem treinamento. }\end{array}$ \\
\hline
\end{tabular}

Fonte: Elaborado pelos autores.

Após a leitura crítica e a sistematização das informações, agruparam-se os manuscritos em 3 categorias: a) Produção do cuidado à mulher em situação de violência sexual; b) Ensino e serviço: campos (des)legitimados para atenção à mulher em situação de violência sexual; e c) Limites na qualificação profissional para atendimento às mulheres em situação de violência sexual.

\section{Produção do cuidado à mulher em situação de violência sexual}

Refletir sobre o cuidado à mulher em situação de violência sexual remete a pensar sobre o que é cuidado, quem cuida e por que cuida e, sob essa perspectiva de interpretação, o cuidado tem diferentes (e às vezes complexos) significados.

Contudo, convém salientar que o cuidado deve envolver a integração de saberes e práticas de diferentes campos do conhecimento, complementando e apresentando intersecções para a promoção da saúde de mulheres em situação de violência, que se revelam seres complexos, cuja saúde é alcançada diante da atenção das necessidades despertadas em um corpo constituído por fatores biológicos, psicológicos, sociais e culturais.

Um estudo desenvolvido no Canadá com residentes médicos, versando sobre a atenção à saúde da mulher com sinais de violência, identificou que os profissionais priorizavam o tratamento da lesão como único direcionamento clínico, sem exploração da etiologia. 0 cirurgião estava atento apenas às questões cirúrgicas, não identificando a violência como um problema e não se considerando como melhor provedor para o cuidado ampliado $0^{13}$.

Esses achados são semelhantes a um estudo realizado na Turquia que, ao avaliar o conhecimento dos enfermeiros quanto à capacidade de reconhecer os sinais de abuso físico em mulheres, declarou que os 


\section{...refletir estratégias destinadas a envolver diversos segmentos para a resolução do problema...}

profissionais não têm adotado o cuidado integral em seu fazer diário, devido às pressões de seu trabalho duro no serviço de saúde ${ }^{14}$.

Destarte, compreende-se que, para a prestação de cuidado, faz-se necessário que o profissional esteja habilitado a atender às peculiaridades do indivíduo, pois se verificam situações em que a queixa, para ser solucionada, requer ampliação da atenção com uma visão do todo, que se traduz em reconhecer o indivíduo inserido em um contexto social.

Uma investigação realizada no Município de Fortaleza, cujo objetivo era analisar a utilização de protocolos na atenção à saúde de mulheres em situação de violência sexual sob a ótica de profissionais, constatou que o atendimento prioritário era imputado ao assistente social, associando sua formação ancorada nos direitos sociais ${ }^{15}$.

Outro estudo, desenvolvido em uma unidade da Estratégia Saúde da Família (ESF) localizada no Município de João Pessoa, que buscou identificar os diferentes elementos do processo de trabalho na assistência à saúde da mulher em situação de violência de gênero, apontou que a resolução das questões relacionadas a essa mulher estaria direcionada aos psicólogos ${ }^{16}$, reconhecido como profissional qualificado para solucionar situações complexas ${ }^{16,17}$.

Considera-se que esses posicionamentos refletem a fragilidade no campo da formação profissional, que, apesar do compartilhamento de campos de atuação, não proporciona integração de conteúdos e práticas colaborativas capazes de reduzir a fragmentação da atenção à saúde em situações complexas.

Desse modo, deve-se modificar o processo de trabalho para um fazer e agir em rede, desarticulando os espaços demarcados e isolados de categorias profissionais ${ }^{15-18}$. Os desdobramentos das ações precisam refletir estratégias destinadas a envolver diversos segmentos para a resolução do problema e não uma perda de controle da atenção prestada ao indivíduo $0^{15,17-19}$.

Diante do exposto, sugere-se a participação de todos os profissionais do serviço de saúde ${ }^{15}$ para uma atuação interdisciplinar, que possibilitará o desvelamento de conhecimentos específicos para um saber comum sobre as diversas expressões da violência sexual contra a mulher ${ }^{17,20}$.

\section{Ensino e serviço: campos (des)legitimados para atenção à mulher em situação de violência sexual}

A inclusão do tema violência na formação profissional em saúde ocorreu por volta da década de 1990 no Brasil. Desde então, o governo vem contribuindo para ampliar a discussão desse tema por meio de dispositivos sociais e da produção de materiais educativos.

Dentre estes se destacam cursos de formação a distância, normas técnicas e manuais educativos, com o propósito de minimizar o déficit de conhecimento da formação, que ainda não incluía nos conteúdos curriculares situações que problematizassem a realidade sociossanitária dos municípios brasileiros ${ }^{15}$.

Todavia, estudos revelam que a formação não expressa preocupação com a abordagem do tema violência contra a mulher ${ }^{20,21}$, pois este ainda sofre influência do modelo biomédico, centrado na doença ${ }^{15,19}$. 0 s agravos à saúde da população brasileira, que vêm tornando-se cada vez mais comuns, fogem da expertise dos profissionais, que não sabem quais condutas adotar diante desses $\operatorname{casos}^{15,19,20}$.

Investigação realizada com profissionais da saúde das unidades da ESF de um distrito sanitário no Município de Salvador revelou que a abordagem do tema na academia ocorreu de modo superficial e pontual e que o despreparo na formação profissional repercute em dificuldades na atenção às mulheres em situação de violência ${ }^{20}$.

Desse modo, considera-se a necessidade de qualificação com foco na formação profissional, em nível de graduação e pós-graduação, contemplando os interesses sociais e de saúde da população ${ }^{20}$, principalmente no tocante à violência sexual contra a mulher e seus diferentes desdobramentos ${ }^{15,20}$.

Verifica-se, ainda, que a fragilidade da qualificação profissional ${ }^{15,19,22,23}$ para esse tipo de atuação é intensificada pela falta de treinamento no contexto dos serviços ${ }^{15,16,20,21}$, contribuindo para uma 
assistência sem preparo e envolvimento, limitandose ao tratamento da lesão física ${ }^{15,16,18}$.

Nesse sentido, denota-se ser importante repensar a formação profissional ${ }^{15,23}$, garantindo a inserção do tema violência sexual contra a mulher no currículo mínimo, baseada na necessidade da comunidade ${ }^{24}$, uma vez que, as instituições de Ensino Superior (IES) assumem o importante papel de ampliar a discussão nos espaços acadêmicos e viabilizar a compreensão da construção desigual entre os gêneros ${ }^{20}$.

A formação também deve ser articulada aos campos de trabalho, no sentido de contribuir para ampliar a discussão sobre a violência sexual. A integração com a academia fortalece o processo de formação e possibilita desvelar e transformar a realidade, o que pode decorrer das atividades extracurriculares e da aproximação direta do aluno nos campos de prática.

Como modo de gerenciar a formação profissional para garantir o atendimento adequado às vítimas de violência, um estudo desenvolvido com profissionais da ESF em um município de Santa Catarina identificou a importância de parcerias com as universidades para a realização de práticas durante o processo de trabalho ${ }^{17}$.

A educação permanente em saúde se apresentou como outra ferramenta relevante ao incitar práticas profissionais transformadoras e viabilizar a adoção do contexto ampliado para a atenção integral à saúde ${ }^{15-18}$. Dentre as estratégias, aborda-se como exemplo as discussões de caso em reunião de equipe, que envolve todos os profissionais para a construção de um plano de cuidados. Isso possibilita o desenvolvimento de competências e de habilidades interpessoais para incorporar subjetividades inerentes ao cuidado humanizado, com vistas a atender às necessidades de saúde, considerando a transversalidade da violência e sua ocorrência como perda do completo direito humano à saúde ${ }^{17}$.

\section{Limites na formação profissional para atendimento às mulheres em situação de violência sexual}

A violência contra a mulher se manifesta como um fenômeno complexo e multidimensional, que implica a dificuldade dos profissionais em conduzir situações que a envolvam, uma vez que é notório o déficit de conhecimento quanto às condições clínico-biológicas ${ }^{15,16,18,19,22}$, aos aspectos sociais e psicológicos do indivíduo vitimizado ${ }^{18-20,22}$, às resistências culturais para abordar a questão com as

\section{A violência contra a mulher se manifesta como um fenômeno complexo e multidimensional...}

pacientes, à priorização do modelo biologicista ${ }^{18,19}$, à alta rotatividade dos profissionais da $\operatorname{ESF}^{17,20}$ e ao reconhecimento da violência sexual em uma lesão física e superficial ${ }^{18,22}$.

A dificuldade dos profissionais da saúde para enfrentar a violência em sua prática cotidiana ${ }^{15,17,19,20}$ decorre de não perceberem determinados episódios como situações de violência ${ }^{15,18,20,22}$ e do receio de sinalizar para a equipe os casos identificados, evoluindo para um atendimento esvaziado e com descontínuo tratamento. Isso acarreta uma segunda violência nos atendimentos, os quais deveriam acolher e apontar o caminho para a proteção, com garantia de direitos ${ }^{18,22,25}$.

Esse fato se relaciona à baixa demanda explícita de atendimentos em decorrência de abusos, motivada pela não identificação do setor saúde, especialmente na ESF, como serviço adequado para a busca de atenção e apoio ou por medo do agressor, vergonha ou naturalização da violência ${ }^{18}$.

Aponta-se que, além da dificuldade de reconhecer casos de violência ${ }^{15,18,20,22}$, às vezes as vítimas não declaram a agressão sofrida ao profissional ${ }^{16,17}$, o que suscita a necessidade de uma experiência profissional distinguir as mulheres vítimas de violência ${ }^{14}$.

Outro impasse apresentado pelos profissionais é que eles não se percebem devidamente preparados para a abordagem e o acompanhamento dos casos de violência ${ }^{15,16,18,20}$, seja com as vítimas, com os agressores, com os familiares e com a própria comunidade, o que inviabiliza uma atenção integral e intersetorial ${ }^{18,25}$, fragmentando a organização do trabalho e instaurando um modelo verticalizado em que cada categoria e especialidade institui seu modo de agir diante de um problema que não é só do setor saúde, mas, sobretudo, social ${ }^{15,18}$.

Essas limitações estão circunscritas na formação profissional 20,23 centrada no paradigma clínicobiológico 20 e na ausência da educação permanente 
na prática dos serviços de saúde ${ }^{15,16}$. A atuação profissional em casos de violência é conduzida pelo improviso, com base nas vivências e na sensibilidade pessoal, comprometendo a qualidade da assistência prestada à saúde da mulher vítima de violência sexual ${ }^{18}$.

Ao considerar a impotência perante os casos de violência se compreende a necessidade dos profissionais em termos de capacitação sobre gênero ${ }^{16,21}$, para proporcionar autonomia e atuação efetiva diante de tais situações, não se restringindo ao encaminhamento, diante da ausência de agravos físicos ${ }^{16,22}$ e/ou atendimento esporádico ${ }^{25,26}$.

Além disso, destaca-se que a formação profissional não é prioridade apenas das IES, mas também deve ser assegurada no serviço de saúde por meio de estratégias que contemplem o tema, capacitando os profissionais já graduados para que reflitam diante da realidade ${ }^{20}$.

\section{CONCLUSÃO}

Os profissionais da saúde que atuam no atendimento à mulher vítima de violência apresentam fragilidades quanto ao reconhecimento dos casos e à prestação de cuidado de modo holístico, a partir das necessidades de saúde do indivíduo, o que implica a atenção a aspectos relativos à resolução de queixas e ao tratamento de agressões físicas, além do encaminhamento das vítimas.

Essas condutas são heranças de uma formação profissional incipiente diante de questões de cunho social e psicológico, influenciada pela valorização de um modelo biologicista, com atenção restrita à queixa-conduta, direcionada pela identificação de uma patologia que requer tratamento, às vezes, medicamentoso. Constata-se que a matriz curricular dos cursos do setor saúde ainda se apresenta povoada por disciplinas cujo escopo se detém no indivíduo e nas patologias inerentes às etapas da vida, deixando um espaço discreto para abordar condições de saúde que remetem a uma perspectiva integral, com necessidade do envolvimento de distintas categorias profissionais.

Quanto às limitações para a produção deste estudo, denota-se a lacuna na literatura diante de questões relativas à formação profissional direcionada à violência sexual contra a mulher, haja vista que a maioria das publicações apontava, principalmente, a caracterização das vítimas de agressão, a quantificação dos distintos casos

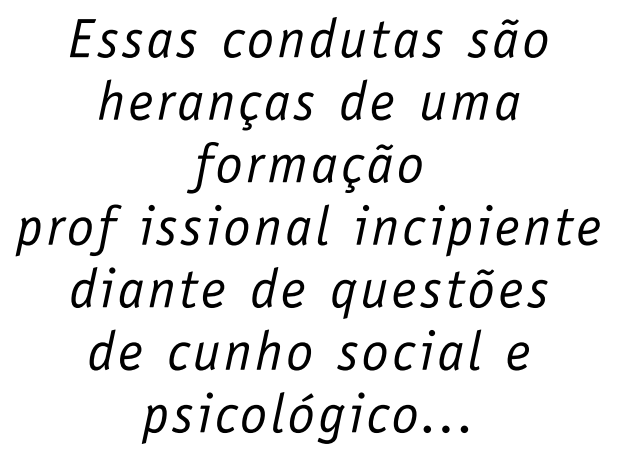

de violência atendidos em serviços de saúde e as dificuldades enfrentadas pelos profissionais da saúde para lidar com situações que envolvam casos de violência.

\section{CONTRIBUIÇÃO DOS AUTORES}

\author{
Francisca Alanny Rocha Aguiar, João Victor \\ Lira Dourado e Raimunda Magalhães da Silva \\ contribuíram com a realização da pesquisa, o \\ delineamento do estudo e a redação e revisão crítica \\ do manuscrito. Ludmila Fontenele Cavalcanti e \\ Luiza Jane Eyre de Souza Vieira contribuíram \\ com o delineamento do estudo e a revisão crítica \\ do manuscrito. Antonio Rodrigues Ferreira Júnior \\ contribuiu com a realização da pesquisa e a redação \\ do manuscrito.
}

\section{REFERÊNCIAS}

1. World Health Organization. Global status report on violence prevention 2014. Geneva: WH0; 2014.

2. Fórum Brasileiro de Segurança Pública. Atlas da Violência 2019. Brasília (DF): Instituto de Pesquisa Econômica Aplicada; 2019.

3. Coelho EBS, Silva ACLG, Lindner SR. Violência: definições e tipologias. Florianópolis: Universidade Federal de Santa Catarina; 2014.

4. Palhoni ARG, Oliveira VJ, Villela LCM, Penna CMM. Representations of women on the quality of life and its relationship with violence against women. Rev Enferm UFPE On Line [serial on the internet]. 2017 [cited 2018 Aug 20];11(4):1701-8. Available from: https://doi.org/10.5205/1981-8963v11i4a15241p1701-1708-2017

5. Fornari LF, Labronici LM. 0 processo de resiliência em mulheres vítimas de violência sexual: uma possibilidade de cuidado. Cogitare Enferm [serial on the internet]. 2018 [cited 2018 Aug 25];23(1):e52081. Available from: http://dx.doi. org/10.5380/ce.v23i1.52081 
6. Russo GHA, Silva HTL, Nogueira JLR, Dantas JGS. Ecos do silêncio: violência sexual denunciada no CREAS Mossoró-RN. Argumentum [serial on the internet]. 2014 [cited 2019 Jan 19];6(1):22339. Available from: https://doi.org/10.18315/ argumentum.v6i1.5921

7. Cavalcanti LF, Moreira GAR, Vieira LJES, Silva RM. Implementação da atenção em saúde às violências sexuais contra as mulheres em duas capitais brasileiras. Saúde Debate [serial on the internet]. 2015 [cited 2019 Jan 20];39(107);107991. Available from: http://dx.doi.org/10.1590/0103$\underline{110420151070381}$

8. Santos CM. Curto-circuito, falta de linha ou na linha? Redes de enfrentamento à violência contra mulheres em São Paulo. Revista Estudos Feministas [serial on the internet]. 2015 [cited 2019 Feb 25];23(2):577-600. Available from: http://dx.doi. org/10.1590/0104-026X2015v23n2p577

9. Penna LHG, Tavares CMM, Dousa ER. The importance of the insert of the thematic "violence against the woman" in the curriculum of nursing. Online Braz J Nurs [serial on the internet]. 2004 [cited 2019 Mar 20];3(2):69-77. Available from: http://www.objnursing.uff.br/index.php/nursing/ article/view/4923/html 794

10. Cavalcanti LF, Silva RM. Violência sexual contra a mulher e a formação profissional na área da saúde. XIII Congresso Mundo de Mulheres; Jul 30Aug 4 2017; Florianópolis, BR. Anais. Florianópolis: Universidade Federal de Santa Catarina; 2017.

11. Soares CB, Hoga LAK, Peduzzi M, Sangaleti C, Yonekura T, Silva DRAD. Revisão integrativa: conceitos e métodos utilizados na enfermagem. Rev Esc Enferm USP [serial on the internet]. 2014 [cited 2019 Jun 10];48(2):335-45. Available from: http://www.scielo.br/scielo.php?script=sci arttext\&pid $=$ S0080-62342014000200335\&lng =en

12. Botelho L, Cunha C, Macedo M. 0 método da revisão integrativa nos estudos organizacionais. Gestão e Sociedade [serial on the internet]. 2011 [cited 2019 Jun 20];5(11):121-36. Available from: https://doi.org/10.21171/ges.v5i11.1220

13. Conn LL, Young A, Rotstein OD, Schemitsch E. "I've never asked one question." Understanding the barriers among orthopedic surgery residents to screening female patients for intimate partner violence. Can J Surg [serial on the internet]. 2014 [cited 2018 Aug 10];57(6):121-7. Available from: https://doi.org10.1503/cjs.000714

14. Yildiz T, Selimen D, Dogan D. A study comparing the pre- and post-training knowledge of emergency department nurses in Turkey for the diagnosis of physically abused women. J Fam Violence [serial on the internet]. 2014 [cited 2018 Aug 17];29(5):519-
525. Available from: https://link.springer.com/ article/10.1007/s10896-014-9608-3

15. Vieira LJES, Silva ACF, Moreira GAR, Cavalcanti LF, Silva RM. Protocolos na atenção à saúde de mulheres em situação de violência sexual sob a ótica de profissionais de saúde. Ciênc Saúde Colet [serial on the internet]. 2016 [cited 2018 Aug 20];21(12):3957-65. Available from: http://dx.doi. org/10.1590/1413-812320152112.15362015

16. Almeida LR, Silva ATMC, Machado LS. 0 objeto, a finalidade e os instrumentos do processo de trabalho em saúde na atenção à violência de gênero em um serviço de atenção básica. Interface Comun Saúde Educ [serial on the internet]. 2014 [cited 2018 Sep 8];18(48):47-60. Available from: http://dx.doi. org/10.1590/1807-57622014.0560

17. Gomes NP, Erdmann AL, Stulp KP, Diniz NMF, Correia $C M$, Andrade SR. Cuidado às mulheres em situação de violência conjugal: importância do psicólogo na Estratégia de Saúde da Família. Psicol USP [serial on the internet]. 2014 [cited 2018 Sep 20];25(1):63-9. Available from: http://dx.doi.org/10.1590/S010365642014000100007

18. Porto RTS, Bispo Júnior JP, Lima EC. Violência doméstica e sexual no âmbito da Estratégia de Saúde da Família: atuação profissional e barreiras para o enfrentamento. Physis (Rio J) [serial on the internet]. 2014 [cited 2018 Sep 25];24(3):787-807. Available from: http://dx.doi.org/10.1590/S0103$\underline{73312014000300007}$

19. Dourado SM, Noronha CV. Marcas visíveis e invisíveis: danos ao rosto feminino em episódios de violência conjugal. Ciênc Saúde Colet [serial on the internet]. 2015 [cited 2018 0ct 27];20(9):2911-20. Available from: http://dx.doi.org/10.1590/141381232015209.19012014

20. Cordeiro KCC, Santos RM, Gomes NP, Melo DS, Mota RS, Couto TM. Formação profissional e notificação da violência contra a mulher. Rev Baiana Enferm [serial on the internet]. 2015 [cited 2018 0ct 17];29(3):209-17. Available from: http://dx.doi. org/10.18471/rbe.v29i3.13029

21. Santos J, Andrade RL, Reis LA, Duarte SFP. Conhecimento de enfermeiras em unidades de saúde sobre a assistência à mulher vítima da violência. Rev Baiana Enferm [serial on the internet]. 2014 [cited 2018 Nov 6];28(3):260-70. Available from: http://dx.doi.org/10.18471/rbe.v28i3.9255

22. Bezerra JF, Silva RM, Cavalcanti LF, Nascimento JL, Vieira LJES, Moreira GAR. Conceitos, causas e repercussões da violência sexual contra a mulher na ótica de profissionais de saúde. Rev Bras Promoç Saúde [serial on the internet]. 2016 [cited 2018 Nov 17];29(1):51-9. Available from: http://dx.doi. org/10.5020/18061230.2016.p51 
23. Schaffir J, Waddell V, Watson D, Way D. National survey on psychosocial obstetrics and gynecology curriculum in US medical schools. J Psychosom Obstet Gynaecol [serial on the internet]. 2014 [cited 2018 Nov 20];35(2):37-41. Available from: https://www.ncbi.nlm.nih.gov/pubmed/24766531

24. Baragatti DY, Audi CAF, Melo MC. Abordagem sobre a disciplina violência em um curso de graduação em enfermagem. Rev Enferm UFSM [serial on the internet]. 2014 [cited 2018 Nov 25];4(2):470-7. Available from: http://dx.doi.org/10.5902/2179769211265

25. Silva EB, Padoin SMM, Vianna LAC. Mulher em situação de violência: limites da assistência. Ciênc Saúde Colet [serial on the internet]. 2015 [cited 2018 Dec 5];20(1):249-58. Available from: http:// dx.doi.org/10.1590/1413-81232014201.21202013

26. Nielson MH, Strong L, Stewart JG. Does sexual assault nurse examiner (sane) training affect attitudes of emergency department nurses toward sexual assault survivors? J Forensic Nurs [serial on the internet]. 2015 [cited 2018 Dec 15];11(3):13743. Available from https://insights.ovid.com/ article/01263942-201507000-00004

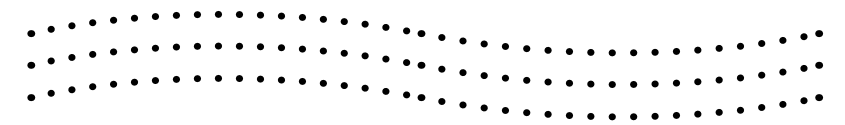

$\cdots \cdots$

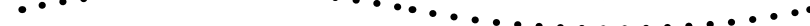
$\ldots \ldots \ldots \ldots \ldots \ldots \ldots \ldots \ldots \ldots \ldots \ldots \ldots \ldots \ldots \ldots \ldots \ldots \ldots \ldots$ $\ldots \ldots \ldots \ldots \ldots \ldots \ldots \ldots \ldots \ldots$ $\ldots \ldots \ldots \ldots \ldots \ldots \ldots \ldots \ldots \ldots$
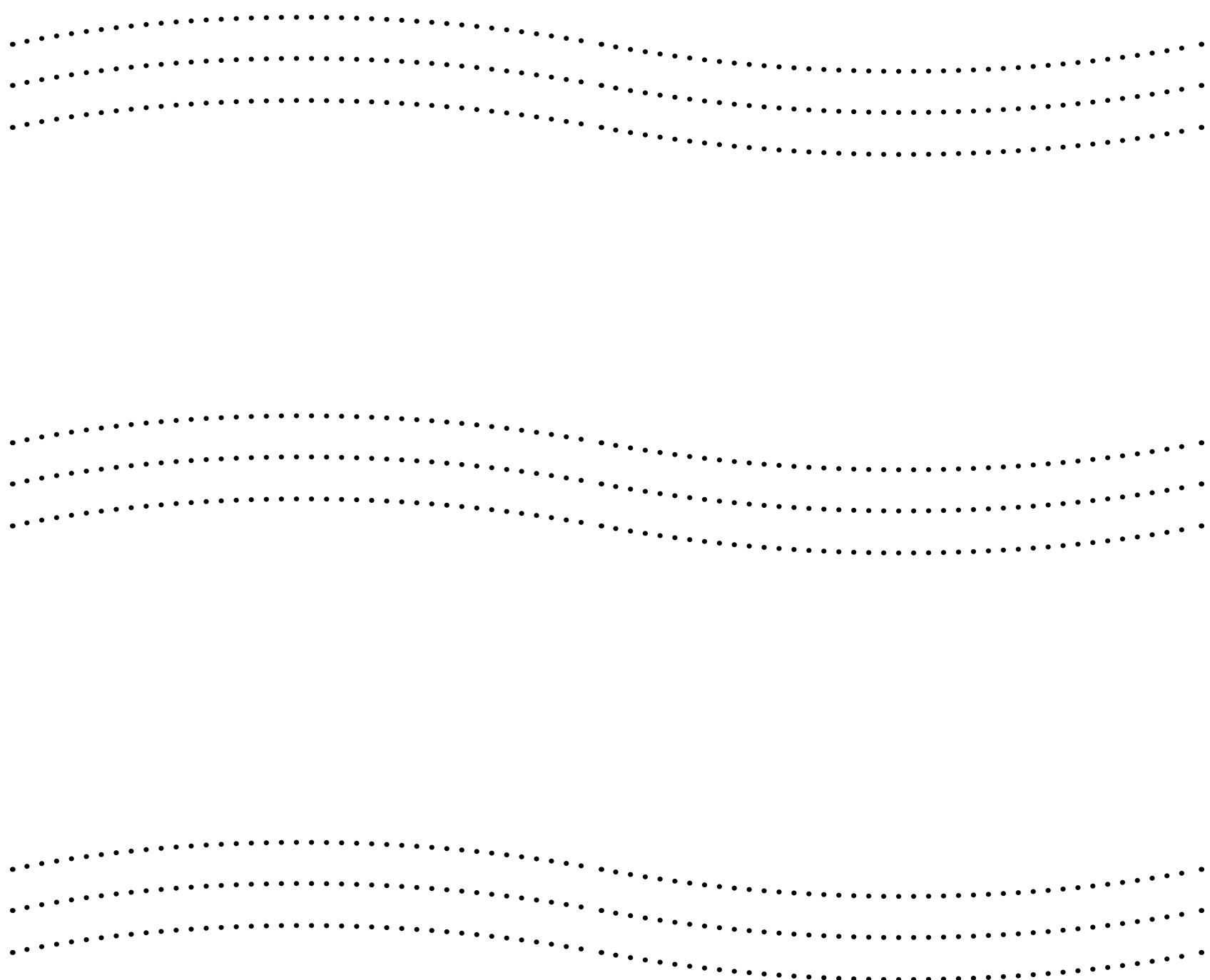